\title{
Industrial Automatic Assembly Technology Based on Machine Vision Reco- gnition
}

Shiqian Xiang

School of Art, Gansu University of Political Science and Law, Lanzhou, Gansu 730070, China. E-mail: xiang_shiq@126.com

With the rapid development of science and technology, the means of industrial production have become more diversified and intelligent, and the development of new means of industrial production has become an increasingly important research topic. Therefore, the automatic assembly technology was studied, taking machine vision system as the main research subject in this study. An automated assembly model of industrial technology based on machine vision recognition was established, parameters, such as the part positioning parameter, assembly time, the number of parts wrongly assembled, the number of parts missing, and the assembly qualification rate were obtained, and the corresponding experimental conclusions were obtained. Moreover, it was compared with the traditional manual assembly technology, and it was found that the automatic assembly technology based on machine vision recognition had better performance and more remarkable experimental results compared with the traditional manual assembly technology, and the traditional manual assembly technology needed continuous modification and optimization. This work provides a new route for automatic assembly technology in industrial technology.

Keywords: Machine vision, Recognition, Industrial technology, Automated assembly

\section{Introduction}

With the rapid development of society and economy, the requirements and standards for industrial production technology are becoming higher and higher. Therefore, it is necessary to combine some new technologies, such as machine vision technology, genetic algorithm, and radio frequency identification technology. Combining these new technologies can effectively improve the efficiency of industrial production and enhance the quality of industrial products to further improve and perfect the industrial production mechanism [1]. In response to this problem, many experts and scholars have put forward their views. Chauhan and Surgenor [2] proposed a machine visionbased fault detection method for automatic assembly machines and found that the method had less training and processing time and could detect faults faster than the spatiotemporal volume (STV) method. They also proposed a method of fault detection and classification based on machine vision for automatic assembly machines, which verified that automatic assembly machines could run continuously to achieve high productivity by using the method [3]. Zorcolo et al. [4] considered that the effective lighting of the working area constituted the key aspect of optimizing the performance of automatic assembly technology and proposed a lighting solution for machine vision applications for automatic assembly operations [4]. Chauhan and Surgenor [5] proposed a Machine Vision Inspection
(MVI) system for performance evaluation in automatic assembly machine fault detection. Three MVI methods based on computer vision technology were applied, which verified that the design of an automatic assembly machine could run all-weather to maintain high production efficiency. Odenthal et al. [6] proposed an enhanced vision system for human-computer collaboration in a cognitive automation assembly unit and designed an experimental assembly unit in which the robot performed coordinated pickup and placement operations with small workpieces. Taking the machine vision system as the research subject, an automatic assembly model of industrial technology based on machine vision recognition was established, and the experimental results and conclusions were obtained. This study provides a new model for automatic assembly technology in industrial technology.

\section{Machine vision}

Machine vision is a comprehensive technology [7] that can perceive the position and shape of objects in the surrounding environment [8]. Its main working principle [9] is to obtain the relevant information of images by using cameras, process the information of these images by using processors and obtain the relevant recognition results. Machine vision uses different types of cameras [10]. It converts the target object from light signals to image analog electrical signals and then converts these image analog electrical signals into 
digital signals [11]. The processor obtains the recognition of information by using digital signals.

The main components of the machine vision system [12] include light source system, camera, image acquisition card, servo motor, etc. The selection of light source system needs to consider various conditions [13]; the main function of a camera is to capture images and process and transmit them [14]; an image acquisition card is the link and medium of image acquisition and processing [15]; servo motor is a kind of motion control hardware equipment. The main features of a machine vision system include automation and flexibility. Fig. 1 is a schematic diagram of the main working principles of a machine vision system.

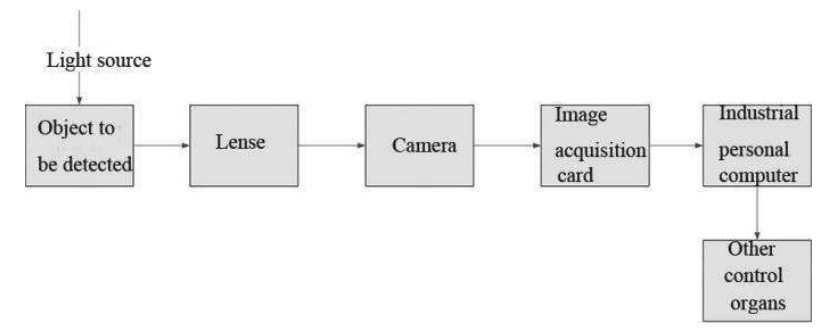

Fig. 1 The main working principle of the machine vision system

Machine vision has been widely used in various fields, including the industrial field, medical field, agricultural field, military field, scientific research field, etc. because of its advantages [16]. In this study, the application of machine vision systems in the industrial field was mainly analyzed. The feasibility and practicability of the machine vision system in automatic assembly technology are mainly studied, taking the machine vision system as the main research subject.

\section{Research on automatic assembly technol- ogy}

The problem of automatic assembly in industrial production was mainly studied, and an automatic assembly model of industrial technology based on machine vision recognition was established.

(1) The machine vision system is initialized, the platform motion is returned to zero, and the user is prompted to place the parts to be assembled on the system screen.

(2) When the parts are clamped to the measuring position, whether the parts are clamped correctly is determined. If the parts are clamped correctly, the features of the parts are measured, and whether exert part assembly task is determined; the parts which are not clamped correctly are remeasured.

(3) Automated assembly operation is carried out on the parts entrusted with the assembly task, and then the assembly results are detected to complete the assembly task of the parts.

\subsection{Image preprocessing and feature extraction}

In machine vision recognition technology, the camera needs to be calibrated first. Therefore, the general calibration method in stereo vision was used to calibrate the camera to understand the precise values of various parameters in the model [17] and locate and analyze the objects that need to be recognized more accurately. In addition, after image acquisition by using camera equipment, it is necessary to pre-process the required image [18]. In this study, histogram binarization was used for image pre-processing, such as image segmentation. Moreover, an iterative algorithm was used to determine the optimal threshold, and the detection and extraction of image edge features were realized by using the Laplace operator, providing preconditions and conditions for the final and effective completion of automatic assembly.

In the binarization of histogram, the histogram is a statistic [19], which shows the global information of an image. In this experiment, a histogram can display the relevant information of the parts to be assembled. It is assumed that $v_{\text {represents the grey value, }} p\left(v_{a}\right)$ represents the probability density of the grey value, $C$ stands for the number of pixels with grey value ${ }^{v}$, and $D$ represents the total number of pixels on the image, the following formula is obtained:

$$
p\left(v_{a}\right)=\frac{C}{D},
$$

The Laplace operator $\Delta^{2}$ adopted in this paper is a form of second-order difference, and $3 \times 3$ mask $e$ is often used. The domains of 4-neighborhood and 8neighborhood are defined as:

$$
e=\left[\begin{array}{ccc}
0 & 1 & 0 \\
1 & -4 & 1 \\
0 & 1 & 0
\end{array}\right] \quad e=\left[\begin{array}{ccc}
1 & 1 & 1 \\
1 & -8 & 1 \\
1 & 1 & 1
\end{array}\right],
$$

\subsection{Image recognition and positioning}

After image pre-processing and other operations mentioned above, it is necessary to recognize the target object from the segmented image, calculate the central coordinates of each object and determine the direction of the object, identify and locate the image, and finally ensure that the machine vision system in this experiment can quickly and accurately grasp the parts and complete the task of automatic assembly.

In this study, the images of parts that need to be assembled are processed by matching and recognition through feature parameters including perimeter $L$, rectangularity $C$ and circularity $Q$. Perimeter $L$ represents the length of the outer boundary of all pixels around a region, which can be obtained by chain code. 
It is supposed that $S$ represents the area of the image area, $S_{0}$ represents the area of the part object, and $S_{C}$ represents the area of the smallest outer rectangle. Then the following formula can be obtained:

$$
\begin{gathered}
C=\frac{S_{0}}{S_{C}}, \\
Q=\frac{4 \pi S}{L^{2}},
\end{gathered}
$$

Moreover, the Hausdorff distance calculation method was used to locate the parts that need to be assembled. The main content of Hausdorff distance calculation method is to define the maximum and minimum distance on two-point sets. It is assumed that the set of pixels of the image under test is $L=\left\{l_{1}, l_{2}, l_{3}, \cdots l_{p}\right\}$, the set of feature points of the template is $N=\left\{n_{1}, n_{2}, n_{3}, \cdots n_{q}\right\}, h(N, L)$ represents the distance between the template and image, $h(L, N)$ represents the distance between the image and template, then the formula of the bidirectional Hausdorff distance $H(N, L)$ between the template and image can be obtained:

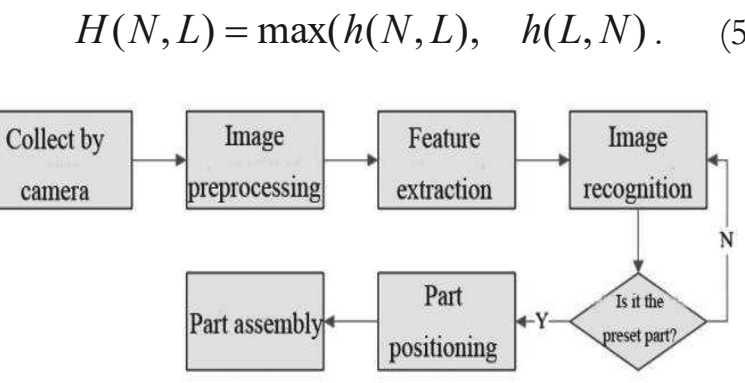

Fig. 2 The flow of part assembly based on machine vision

As shown in Fig. 2, the processed parts are identified and positioned using the industrial camera to realize parts assembly automation. The operation flow is as follows.

(1) The image of the part that needs to be assembled is collected by industrial cameras.

(2) After preprocessing the collected image, the original image is transformed into a grey image by the gamma equalization normalization to reduce the "noise" in the original image and the influence of noise on the recognition, positioning, and assembly of parts.

(3) Features are extracted from the preprocessed part image according to the method described above, and then the image is segmented and recognized according to the extracted image features.

(4) According to the image recognition results, whether the part under the camera is the preset part to be assembled is determined. If not, the part will not be processed, and the recognition of the next part will continue; if it is, the part will be positioned, including calculating the centre coordinates of the object and determining the direction of the object.

(5) The parts are assembled according to the type of parts and positioning results.

\section{Simulation experiment}

\subsection{Experimental methods and parameters}

In this study, a machine vision system was taken as the main research subject, and its application in automatic assembly technology was studied. Seven hundred spherical parts with through holes and $V$ grooves were selected for simulation experiments. The automatic assembly operation was carried out through a machine vision system. The positioning deviation degree of $\mathrm{V}$ grooves and valve spool orifices in the assembly process was analyzed concretely. Parameters, such as the part positioning parameter, assembly time, the number of parts wrongly assembled, the number of parts missing, and the qualification rate of the assembly was obtained. The performance of automatic assembly of the machine vision system was measured using the parameters, and the corresponding experimental conclusions were obtained. Moreover, it was compared with the traditional manual assembly technology [20], and corresponding experimental conclusions were obtained for reference.

In the above experiment, relevant parameters of the camera used for machine vision recognition are as follows. It was an MV-HS series high-speed industrial camera produced by Microvision company. The specific model, highest resolution, optical size, and pixel size of the camera were MV-HS900GM/C, $4096 \times 2160,1^{\prime \prime}$, and $3.45 \mu m \times 3.45 \mu m$ respectively. The type of sensor was Complementary Metal Oxide Semiconductor (CMOS). Its exposure mode was global exposure. Its largest framerate was $13 \mathrm{fps}$.

\subsection{Experimental results}

(1) Automatic assembly process

1. In this simulation experiment, a machine vision system was selected to study the automatic assembly of parts to be assembled, and the related information of parts to be assembled was perceived and acquired. Fig. 2 is the display diagram of the machine vision system to be used in this simulation experiment.

2. The selected parts to be assembled were spherical parts with through holes and $\mathrm{V}$ grooves. In the process of automatic assembly of parts, the positioning experimental parameters of $\mathrm{V}$ grooves and valve spool orifices were analyzed to study the experimental performance of the automatic assembly. Fig. 3 is a display of the parts to be assembled in this simulation experiment. 


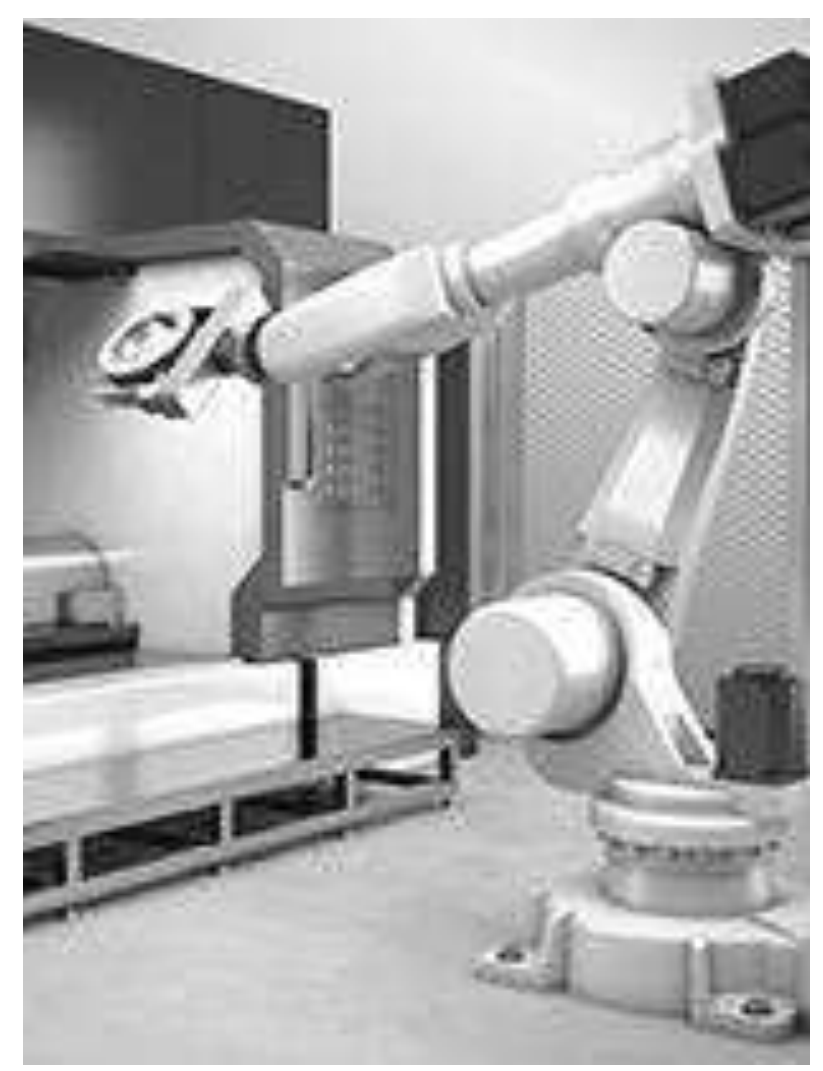

Fig. 2 Display of macbine vision systém

3. Through pretreatment and positioning of assembly parts, the direction and position of parts were determined, the automatic assembly task of spherical parts was finally completed, and the final experimental results were obtained. The image of the assembled part is shown in Fig. 4.

(2) Parts assembly positioning

Based on machine vision technology, assembly positioning experiments were carried out six times for some parts assembled. The offset values of the V groove and spool orifice were obtained, as shown in Tab. 1.

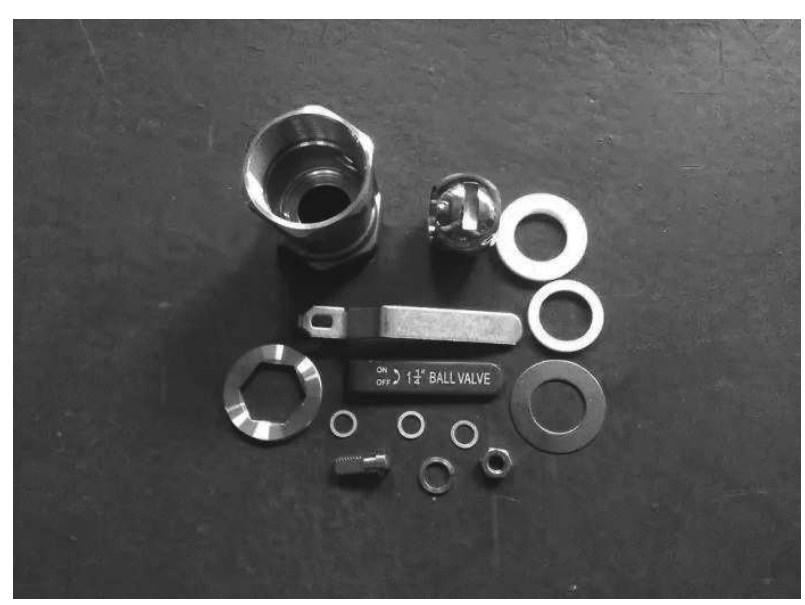

Fig. 3 Display of parts to be assembled

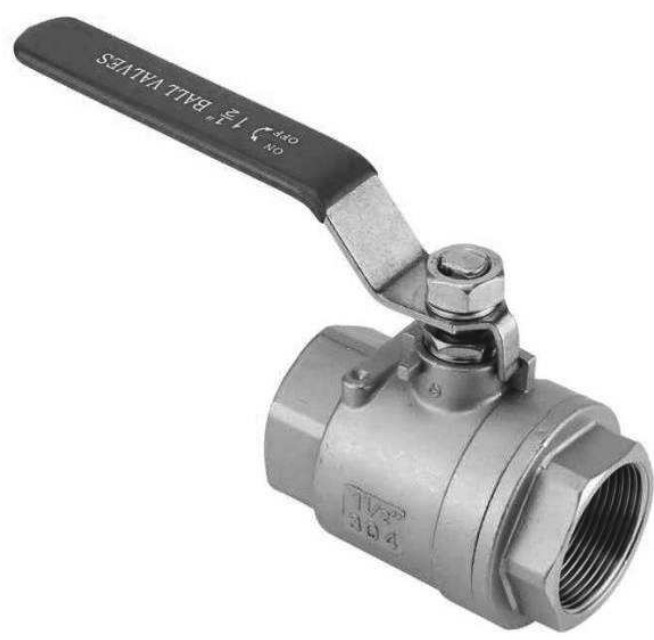

Fig. 4 The assembled part

Tab. 1 The experimental positioning parameters based on the macbine vision system

\begin{tabular}{|l|l|l|l|l|}
\hline $\begin{array}{l}\text { Number of ex- } \\
\text { periments }\end{array}$ & $\begin{array}{l}\text { X-direction offset of } \\
\text { V groove } \\
\text { (pixel) }\end{array}$ & $\begin{array}{l}\text { Y-direction offset of } \\
\text { V groove } \\
\text { (pixel) }\end{array}$ & $\begin{array}{l}\text { X-direction offset of } \\
\text { spool orifice } \\
\text { (pixel) }\end{array}$ & $\begin{array}{l}\text { Y-direction offset of } \\
\text { spool orifice (pixels) }\end{array}$ \\
\hline 1 & 0 & 0 & 1 & 0 \\
\hline 2 & 1 & 0 & 0 & 0 \\
\hline 3 & 0 & 2 & 0 & 0 \\
\hline 4 & 0 & 0 & 0 & 2 \\
\hline 5 & 0 & 1 & 0 & 0 \\
\hline 6 & 1 & 0 & 0 & 0 \\
\hline
\end{tabular}

Tab. 1 shows the experimental positioning parameters of assembly parts obtained based on the machine vision system. It can be seen that in the six parts assembly experiments, the spool orifice deviated by one pixel at the $\mathrm{x}$ direction in the first experiment; the $\mathrm{V}$ groove deviated by one pixel at the $\mathrm{x}$ direction in the second experiment; the $\mathrm{V}$ groove deviated by two pixels at the $\mathrm{Y}$ direction in the third experiment; the spool orifice deviated by two pixels at the $\mathrm{Y}$ direction in the fourth experiment; the $V$ groove deviated by one pixel at the $\mathrm{Y}$ direction in the fifth experiment; the $\mathrm{V}$ groove deviated by one pixel at the $\mathrm{X}$ direction in the sixth experiment. It can be seen that the maximum value of V-groove offset and spool orifice offset was two pixels, the offset value was not very large, and the offset range was small. Therefore, it can be concluded that the machine vision system had a high accuracy in assembling the assembled parts, and the experimental 
results were obvious.

(3) Parts assembly time

The assembly time of some parts in the machine vision system was selected randomly as data, and it was compared with the traditional manual assembly technology, as shown in Fig. 5.

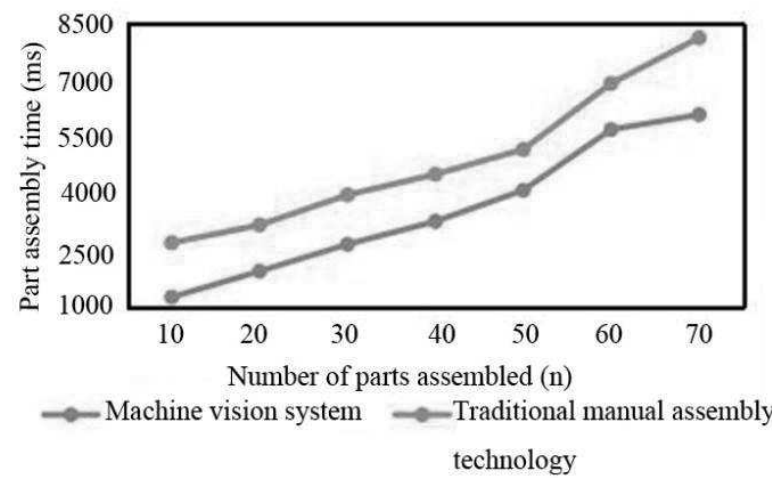

Fig. 5 Comparison of assembly time between the two methods

Fig. 5 shows that the comparative analysis of part assembly time of two different methods. As can be seen from Fig. 5, with the increase of the number of assembly parts, the assembly time of parts also showed a growing trend. When the number of assembled parts was 10 , the assembly time of parts based on the machine vision system reached the minimum value of $1259 \mathrm{~ms}$, and the assembly time of parts based on the traditional manual assembly technology reached the minimum value of $2698 \mathrm{~ms}$. When the number of assembled parts was 70 , the assembly time of parts based on the machine vision system reached the maximum value of $6098 \mathrm{~ms}$, and the assembly time of the parts based on the traditional manual assembly technology reached the maximum of $8122 \mathrm{~ms}$. Therefore, compared with the traditional manual assembly technology, the machine vision system had shorter assembly time, higher assembly speed, and better performance of part automatic assembly, while the traditional manual assembly technology needed continuous improvement and optimization.
(4) Qualification rate of parts assembly

In the assembly technology of industrial production, the qualification rate of part assembly is an important parameter to measure the system performance. The qualification rate of part assembly of the machine vision system was compared with that of traditional manual assembly technology, as shown in Fig. 6.

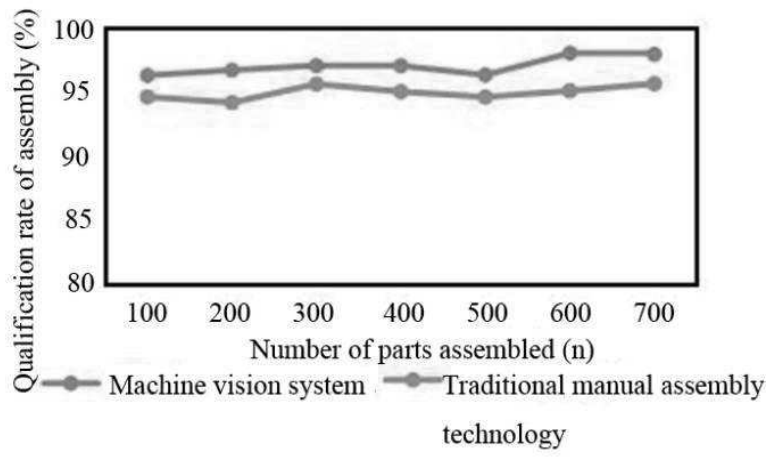

Fig. 6 Comparison of the qualification rate of parts assembly between the two methods

Fig. 6 shows the comparative analysis of the qualification rate of parts assembly of two methods. It can be seen from Fig. 6 that with the increase of the number of parts assembled, the qualification rate of parts assembled in two different ways fluctuated up and down. In the automatic assembly process based on machine vision system, when the number of assembly parts was 600 , the qualification rate was the maximum, $97.96 \%$; when the number of assembly parts was 100 , the qualification rate was the minimum, $96.23 \%$. In the assembly process based on the traditional manual assembly technology, when the number of assembly parts was 700 , the qualification rate was the maximum, $95.59 \%$; when the number of assembled parts was 200 , the qualification rate was the minimum, $94.12 \%$. Therefore, compared with the traditional manual assembly technology, the qualification rate of the machine vision system was higher, and the experimental effect was more remarkable.

Tab. 2 Comparison of the qualification rate of parts assembly between the two methods

\begin{tabular}{|c|c|c|c|}
\hline & Assembly qualification & Assembly error & Assembly missed \\
\hline Machine vision system & $685 / 700$ & $9 / 700$ & $6 / 700$ \\
\hline $\begin{array}{c}\text { Traditional manual as- } \\
\text { sembly technology }\end{array}$ & $669 / 700$ & $20 / 700$ & $11 / 700$ \\
\hline
\end{tabular}

Tab. 2 shows the comparative analysis of the part assembly effect of two different methods. It can be seen from Tab. 2 that when the total number of parts assembled was 700, 685 parts were qualified in the assembly based on the machine vision system, nine parts were wrongly assembled, and six parts were missed; 669 parts were qualified in the assembly based on the traditional manual assembly technology, 20 parts were wrongly assembled, and 11 parts were missed. Compared with the traditional manual assembly technology, the number of qualified parts assembled by machine vision system was larger, and the number of assembly errors and missed parts were small. Therefore, it can be concluded that the machine vision system had a good recognition effect on parts in industrial production and good performance of part automatic assem- 
bly, which is helpful to improve the quality and efficiency of industrial production technology.

\section{Discussion}

The development of industrialization has greatly improved the production speed, especially in the assembly line. In the initial assembly line, each process requires workers to operate, but workers only need to repeat the operation of their processing steps, which puts low requirements for the technical level. The assembly line processing method has indeed improved the production efficiency; however, on the one hand, the repetitive labour with low technical content is not conducive to the physical and mental health of the staff, and on the other hand, it also has a constraint on a large number of labour forces, which could have been engaged in works with more technical values. The emergence of tools, such as robots and manipulators, gradually liberated the labour force. The single and repeated work in the assembly line is very suitable for tools like manipulator that works according to the set program. When the robot or manipulator is working, it usually repeats the work according to the set procedure. However, in the process of assembly line work, one or two parts to be assembled may not be placed according to the regulations, and the rigidly operating mechanical arm is likely to cause misassembly, which will affect the production of the assembly line [21]. Therefore, a monitoring facility is needed to make the manipulator more flexible in assembling parts.

In this study, a machine vision system was designed based on an industrial camera and used in the automatic assembly system. In the whole system, the machine vision system collected the image of the parts to be assembled through the camera, then extracts features from the image, and finally identified the types of parts. The assembly system selected the corresponding assembly operation and positioned the parts at the same time to ensure the accurate position of the parts during assembly [22]. After that, the system was tested. First of all, the positioning test of the machine vision was carried out. The results of the tests showed that the positioning accuracy was high, and the offset was usually one or two pixels. In machine vision, the influence of noise in the image has been reduced as much as possible through preprocessing, but there was still some noise influences. In addition, in the assembly process of parts, vibrations brought by machines would also affect the image quality, resulting in positioning deviation.

Then, the designed system was compared with the traditional assembly method. The content of the comparison included the assembly time of parts and the qualified rate of parts assembly. The experimental re- sults showed that when the number of parts to be assembled increased, both the assembly time of the designed system and the traditional assembly method increased, but the assembly time of the designed system was significantly less under the same assembly quantity. The traditional assembly method adopted manual means. Although the personnel arrangement was easier than the mechanical setting, different workers processed the parts at different speeds, and the same working intensity could not be kept in a long time. The assembly system designed in this study could rapidly recognize and position the parts with the assistance of machine vision and assemble the parts through the mechanical arm; in this process, the working intensity could be maintained to realize more rapid and stable assembly. In terms of the qualified rate of parts assembly, the two kinds of assembly methods were relatively stable under different assembly quantities, and the assembly qualification rate of the system designed in this study was significantly higher. The reason for the high assembly qualified rate is as follows. In the process of manual assembly, workers' actions and attention were different every time of assembly, which made the quality of assembly parts different. In the assembly system, the manipulator could guarantee the consistency of assembly action through the set program, which greatly reduced the instability of assembly quality.

In this study, machine vision technology was combined with automatic assembly technology, and it was used in the automatic assembly of industrial production. The parts to be assembled were recognized and positioned through machine vision to improve the efficiency of automatic assembly. This study provides a reference for improving the efficiency of automatic industrial assembly. The future research direction is to further improve the part recognition and positioning accuracy of machine vision to improve the quality of automatic assembly.

\section{Conclusion}

In this study, the machine vision technology was combined with the automatic assembly technology of industrial production, and then the combined automatic assembly technology was tested and compared with the traditional assembly technology. The results are as follows: (1) in terms of positioning accuracy, the maximum value of $\mathrm{V}$-groove offset and spool orifice offset was two pixels, which was small; (2) in terms of assembly quality, compared with the traditional manual assembly technology, the machine vision system had higher part assembly speed and qualified rate and less number of assembly errors and part missing. The experimental results show that the performance of the automatic assembly system combined with machine vision is better than the traditional assembly technol- 
ogy. This study provides a new path for automatic assembly technology in industrial technology.

\section{References}

[1] CHAWLA, V. K., CHANDA, A. K., ANGRA, S. (2018). A Clonal Selection Algorithm for Minimizing Distance Travel and Back Tracking of Automatic Guided Vehicles in Flexible Manufacturing System. In: Journal of The Institution of Engineers (India): Series C, pp. 1-10.

[2] CHAUHAN, V., SURGENOR, B. (2015). A Comparative Study of Machine Vision Based Methods for Fault Detection in an Automated Assembly Machine. In: Procedia Manufacturing, Vol. 1, pp. 416-428.

[3] CHAUHAN, V., SURGENOR, B. (2017). Fault detection and classification in automated assembly machines using machine vision. In: The International Journal of Advanced Manufacturing Technology, Vol. 90, No. 9-12, pp. 2491-2512.

[4] ZORCOLO, A., ESCOBAR-PALAFOX, G., GAULT, R., SCOTT, R., RIDGWAY, K. (2011). Study of lighting solutions in machine vision applications for automated assembly operations.

[5] CHAuHAN, V., SuRgenOR, B. (2016). Performance evaluation of MVI for fault detection in automated assembly machines. In: 2016 IEEE International Conference on Advanced Intelligent Mechatronics (AIM).

[6] ODENTHAL, B., MAYER, M. P., KABU,B W., SCHLICK, M. (2012). Design and evaluation of an Augmented Vision System for human-robot cooperation in cognitively automated assembly cells. In: International Multi-conference on Systems.

[7] JIANG, H., DUERSTOCK, B. S., WACHS, J. P. (2014). A Machine Vision-Based Gestural Interface for People With Upper Extremity Physical Impairments. In: IEEE Transactions on Systems, Man, and Cybernetics: Systems, Vol. 44, No. 5, pp. 630-641.

[8] CUBERO, S., LEE, W. S., ALEIXOS, N., ALBERT, F., BLASCO, J. (2016). Automated systems based on machine vision for inspecting citrus fruits from the field to postharvest-a review. In: Food and Bioprocess Technology, Vol. 9, No. 10, pp. 1623-1639.
[9] SCHMITT, R., TOBIAS, F., ABBAS, B., ABEL, P., KIMMELMANN, W., KOSSE, P., BURATTI, A. (2016). Automation, Communication and Cybernetics in Science and Engineering 2015/2016. Springer International Publishing.

[10] ARDHY, F., HARIADI, F. I. (2017). Development of SBC-based machine-vision system for PCB board assembly Automatic Optical Inspection. In: 2016 International Symposium on Electronics and Smart Devices (ISESD).

[11] BASKORO, A. S., TANDIAN, R., HAIKAL, EDYANTO, A., SARAGIH, A. S. (2017). Automatic Tungsten Inert Gas (TIG) welding using machine vision and neural network on material SS304. In: International Conference on $A d-$ vanced Computer Science and Information Systems.

[12] COELHO, P. A., TORRES, S. N., RAMÍREZ, W. E., GUTIÉRREZB, P. A., TORO, C. A., SOTO, J. G., SBARBAROA, D. G., PEZOAAB, J. E. (2016). A machine vision system for automatic detection of parasites Edotea magellanica in shell-off cooked clam Mulinia edulis. In: Journal of Food Engineering, Vol. 181, pp. 84-91.

[13] QUIROS, A. R. F., ABAD, A., BEDRUZ, R. A., UY, A. C., DADIOS, E. P. (2016). A genetic algorithm and artificial neural network-based approach for the machine vision of plate segmentation and character recognition. In: International Conference on Humanoid.

[14] DUTTA GUPTA, S., KARMAKAR, A. (2017). Machine vision based evaluation of impact on light emitting diodes (LEDs) on shoot regeneration and the effect of spectral quality on phenolic content and antioxidant capacity in, Swertia chirata. In: Journal of Photochemistry and Photobiology B: Biology, Vol. 174, pp. 162-172.

[15] AL-NAJI, A., CHAHL, J. (2017). Simultaneous Tracking of Cardiorespiratory Signals for Multiple Persons Using a Machine Vision System with Noise Artifact Removal. In: IEEE Journal of Translational Engineering in Health and Medicine.

[16] NOURI-AHMADABADI, H., OMID, M., MOHTASEBI, S., FIROUZ, M. S. (2017). Design, development and evaluation of an online grading system for peeled pistachios equipped with machine vision technology and support vector machine. In: Information Processing in $A g$ riculture, pp. S2214317316300117. 
[17] WANG, X. D. (2015). The precision measurement and assembly for miniature parts based on double machine vision systems. In: International Symposium on Precision Engineering Measurement \& Instrumentation. International Society for Optics and Photonics.

[18] SHI, C., QIAN, J., HAN, S., FAN, B., YANG, X., WU, X. (2018). Developing a machine vision system for simultaneous prediction of freshness indicators based on tilapia (Oreochromis niloticus ) pupil and gill color during storage at $4{ }^{\circ} \mathrm{C}$. In: Food Chemistry, Vol. 243, pp. 134-140.

[19] BALABANTARAY, B. K., DAS, B., BISWAL, B. (2014). Comparison of edge detection algorithms for part identification in a vision guided robotic assembly system. In: Studies in Computational Intelligence, Vol. 543, pp. 183-206.
[20] JIANG, S., LIU, P., FU, D., XUE, Y. M., LUO, W. T., WANG, M. J. (2017). A low-cost rapid upper limb evaluation method in manual assembly line based on somatosensory interaction technology. In: 2015th International Conference on Computer-Aided Design, Manufacturing, Modeling and Simulation (CDMMS 2017). AIP Publishing LLC.

[21] WU, Y., LIN, S., DAI, L., LUO, L. (2017). Dynamic balancing for mixing assembly line based on PSO - GA cooperative optimization. In: $\mathrm{Ma}$ nufacturing Technology, Vol. 17, No. 4, pp. 622628.

[22] LIU, Q., YAN, Y. R., CAO, L. L. (2016). Computer visual measurement technology and algorithm simulation for the assembly of large aircraft parts. In: Manufacturing Technology, Vol. 16, No. 3, pp. 538-543 